Verbrechensdimensionen der beiden totalitären Herrschaftssysteme ${ }^{480}$ - im Vordergrund. Gleichwohl rückten in erster Linie die innen- und außenpolitischen Zustände Frankreichs in das Zentrum seiner Beiträge. ${ }^{481}$ In diesem Zusammenhang beschäftigte er sich in seinen kritischen Untersuchungen mit der Regierungspolitik, ${ }^{482}$ der französischen Kolonialpolitik, ${ }^{483}$ der rechtsradikalen Bewegung von Pierre Poujade ${ }^{484}$ und insbesondere mit der politischen Linken sowie ihren einflussreichen französischen Intellektuellen - wie beispielsweise Jean-Paul Sartre. ${ }^{485}$

\title{
5. Warum hat Franz Neumann nicht im Monat geschrieben? Ein >Negativporträts
}

Im Anschluss an die Porträts von den zehn Autoren des Monat soll in diesem »Negativporträt« versucht werden, eine Antwort darauf zu finden, warum der renommierte NS-Forscher Franz Neumann nicht im Monat geschrieben hat, obwohl er im Laufe der Jahre bis zu seinem Tod im Jahre 1954 prädestiniert gewesen wäre, in der Zeitschrift $\mathrm{zu}$ publizieren. Abgesehen von der persönlichen Lebensgeschichte von Neumann ist damit besonders folgender Sachverhalt gemeint: erstens die wissenschaftlichen Veröffentlichungen, die im Grunde genommen von Anfang an den Totalitarismus als Herrschaftsregime in den Blick nahmen und in denen nach Kriegsende aus einer analytischen Perspektive heraus ein expliziter und impliziter Vergleich zwischen

schlepptenlager durch Verschleppte, in: Der Monat 2 (1950), H. 16, S. 353-356; Vgl. hierzu auch das Kap. III.

480 Obwohl Lüthy sich mit den totalitären Herrschaftssystemen des Nationalsozialismus und des Sowjetkommunismus auch in vergleichender Perspektive auseinandersetzte, war er gleichwohl kein »Anhänger« der klassischen Totalitarismustheorie, weil sie ihm nicht als geeignetes Instrument erschien, die spezifischen Besonderheiten der beiden Regime auf den Begriff zu bringen. Die Veröffentlichungen beispielsweise der ihm persönlich bekannten Theoretiker Hans Kohn und Hannah Arendt wurden von ihm in den 1940- bzw. 1950er-Jahren auch nicht rezipiert. Mitteilung von Lüthy in einem Gespräch mit dem Verfasser in Basel im Juli 2001.

481 Siehe grundsätzlich: Die Vierte Republik. Von Charles de Gaulle zu Henri Queuille, in: Der Monat 1 (1949), H. 8/9, S. 120-134, sowie Die Vierte Republik. Frankreich seit seiner Befreiung, in: Der Monat 1 (1949), H. 10, S. 39-48. Vgl. hierzu Herbert Lüthy, Frankreichs Uhren gehen anders, Zürich u. a. 1954; das Buch entstand aus den beiden zuerst im Monat publizierten »kritischen Essays«. Siehe auch: Vier Jahre später. Frankreichs Uhren gehen immer noch anders, in: Der Monat 10 (1958), H. 114, S. 33-43 (hierbei handelte es sich um das Nachwort der Neuauflage des Buches, das im selben Jahr erschien).

482 Siehe bes. Brief aus Paris: Stabilität oder Stagnation. Die innenpolitische Situation Frankreichs, in: Der Monat 3 (1951), H. 35, S. 465-476; Brief aus Paris: Mendes-France als Liquidator. Frankreichs Rückkehr zur »Realpolitik«, in: Der Monat 7 (1954), H. 73, S. 4-14, sowie Brief aus Paris: Frankreich persönlich? Zu den Memoiren des General Charles de Gaulle, in: Der Monat 9 (1956), H. 98, S. 11-22.

483 Siehe bes.: Das überseeische Frankreich. Ein Kolonialreich in der Krise, in: Der Monat 2 (1949), H. 14, S. 175-186; Brief aus Tunis: Nordafrika wird mündig, in: Der Monat 4 (1952), H. 47, S. 498-515; Ruhm und Ende der Kolonisation, in: Der Monat 9 (1957), H. 106, S. 26-39, sowie Frankreichs Hauptstadt: Algier. Algerien desintegriert das Mutterland, in: Der Monat 10 (1958), H. 118, S. 22-35.

484 Siehe Aufstand in der Wüste. Pierre Poujade und seine »Bewegung«, in: Der Monat 7 (1955), H. 80 , S. 119-127, sowie Heil Poujadolf?, in: Der Monat 8 (1956), H. 90, S. 3-12.

485 Siehe bes. Brief aus Paris: Frankreichs heimatlose Linke, in: Der Monat 2 (1950), H. 19, S. 8-17, sowie Jean-Paul Sartre und das Nichts, in: Der Monat 7 (1955), H. 83, S. 407-414. Vgl. hierzu auch das Kap. III. 
Nationalsozialismus und Sowjetkommunismus gezogen wurde, zweitens seine Mitarbeit für den Regierungsapparat der Vereinigten Staaten im und nach dem Zweiten Weltkrieg, drittens seine persönlichen Kontakte zu einflussreichen Autoren des Monat - wie zum Beispiel Borkenau und Löwenthal - sowie viertens die Tatsache, dass der Emigrant und ehemalige Mitarbeiter des New Yorker Instituts für Sozialforschung seit den späten 1940er-Jahren eine zentrale Rolle bei der Gründung von Forschungseinrichtungen in Berlin spielte und entscheidenden Anteil an der Etablierung der politischen Wissenschaft als »Demokratiewissenschaft« in Deutschland hatte.

Nachdem Neumann am 2. September 1954 bei einem Autounfall in der Schweiz im Alter von 54 Jahren ums Leben gekommen war, hielt Ernst Fraenkel ${ }^{486}$, sein Freund, Kollege und politischer "Kampfgefährte « aus den Jahren der Weimarer Republik, eine Gedenkrede im Jahre 1955 in der Deutschen Hochschule für Politik in Berlin. Im Hinblick auf Neumanns Mitarbeit als »Syndikus« für die Gesamtpartei der SPD seit Mitte 1932 sagte er:

In dieser Eigenschaft hat der Verfassungsanwalt mit verzweifeltem Mut gegen Presseverbote, Versammlungsauflösungen, Verhaftungen, Beamtenentlassungen und ähnliche Willkürakte der Regierungen Papen, Schleicher und Hitler gekämpft. Obgleich politisch gefährdet und als Jude bedroht, blieb Neumann auf seinem Posten, bis am 2. Mai 1933 die SA das im Metallarbeiterhaus in der Alten Jakobstraße gelegene Anwaltsbüro besetzte. Weitere Tätigkeit in Deutschland war sinnlos geworden. Mit den Worten »Mein Bedarf an Weltgeschichte ist gedeckt«, verabschiedete er sich von dem Anwaltspartner und Weggenossen vierzehnjährigen gemeinsamen Strebens und Bemühens. Eine glänzende Karriere war zerstört, der Kampf um ein soziales Arbeitsrecht verloren, der Rechtsstaat zerbrochen, die Demokratie vernichtet [...] Mittellos ist Franz Neumann damals nach England emigriert [...]. ${ }^{487}$

Neumann wurde im Jahre 1900 als Sohn jüdischer Eltern - der Vater war Handwerker und Kleinhändler - im oberschlesischen Kattowitz geboren. Im Alter von 18 Jahren begann er in Breslau mit dem Studium der Rechtswissenschaft, der Philosophie und der Ökonomie und wechselte 1919, nachdem er aktiv als Mitglied einer sozialistischen Studentengruppe 1918 die Barrikadenkämpfe der aufständischen Soldaten und Arbeiter in Leipzig unterstützt hatte, an die Universität Frankfurt a. M. Dort schloss er mit Leo Löwenthal und Fraenkel Freundschaft. Insbesondere die in der Weimarer Republik stark aufgeladenen Themen Arbeitsrecht und Wirtschaftsdemokratie gerieten in den theoretischen Scheinwerfer von Neumann, der sich bereits 1919 für eine Mitgliedschaft in der sich für politische und soziale Reformen einsetzenden SPD und mithin gegen die KPD entschied. 1923 promovierte er mit dem Thema Rechtsphilosophische Einleitung zu einer Untersuchung über das Verhältnis von Staat und Strafe an der Juristischen Fakultät der Frankfurter Universität. Von 1923 bis 1927 folgte im Rahmen der Referendaraus-

486 Wie bereits gesagt, hatte der 1938 in die Vereinigten Staaten emigrierte Ernst Fraenkel mit seiner 1941 in New York erschienenen empirisch ausgerichteten Studie The Dual State eine der ersten wissenschaftlichen Bücher über die Herrschaftsstruktur des >Dritten Reiches v veröffentlicht, die in der analytischen Unterscheidung des Nebeneinanders von »Maßnahmenstaat« sowie »Normenstaat« zu einem Klassiker der NS-Forschung avancierte.

487 Ernst Fraenkel, Gedenkrede auf Franz L. Neumann, in: Ders., Reformismus und Pluralismus. Materialien zu einer ungeschriebenen politischen Autobiographie, Hamburg 1973, S. 168-179, hier S. 175. 
bildung die sowohl politische als auch theoretische Mitarbeit als Assistent von Hugo Sinzheimer, der zu den geistig-politischen Begründern des Weimarer Arbeitsrechts sowie zu einem der Väter der Verfassung der ersten Republik in Deutschland zählte.

Im darauffolgenden Jahr übersiedelte Neumann nach Berlin und eröffnete mit Fraenkel eine Rechtsanwaltskanzlei und wurde zudem Syndikus der Baugewerkschaft sowie anderer Gewerkschaften, die er insbesondere in zahlreichen Prozessen vor dem Reichsarbeitsgericht in Leipzig vertrat. Im Bewusstsein der existenziellen persönlichen Gefahr angesichts der Gefahr einer Machteroberung der »Hitlerbewegung« kämpfte Neumann als "politischer Anwalt« indes auch und vor allem - wie es Fraenkel mitteilte - gegen verschiedene Willkürakte der preußischen Regierung sowie der Reichsregierung. Nachdem der Nationalsozialismus am 30. Januar 1933 tatsächlich an die Schalthebel der staatlichen Macht kam, wurde dem »Überzeugungsrepublikaner« (Helge Pross) Anfang April 1933 die Zulassung als Rechtsanwalt entzogen; als Jude sowie aktiver politischer Gegner der nationalsozialistischen Politik wurde er am 30. April verhaftet. ${ }^{488}$ Nachdem dann die SA unter anderem am 2. Mai 1933 die Gewerkschaftshäuser stürmte und sein Anwaltsbüro besetzte, entschied sich Neumann, Deutschland zu verlassen, und emigrierte - mit seiner damaligen Ehefrau Inge und dem kleinen Sohn Thomas - nach England. ${ }^{489}$ Seitdem fragte er sich persönlich und stand für ihn grosso modo in seinen wissenschaftlichen Arbeiten die Frage im Zentrum, wie der Nationalsozialismus in Deutschland »die Herrschaft übernehmen konnte«. ${ }^{490}$

Neumanns erster im Londoner Exil gegen den Nationalsozialismus veröffentlichter Artikel The Decay of German Democracy aus dem Jahre 1933 zählt zu seinen »Aufklärungsschriften«, mit denen er zum einen seine Gegnerschaft zum totalitären Staat zum Ausdruck brachte sowie zum anderen eine frühzeitige historisch-theoretische Erklärung der Machteroberung der »Hitlerbewegung« lieferte. »Die These dieses Aufsatzes ist«, so Neumann,

daß die nationalsozialistische Revolution eine Konterrevolution der monopolisierten Industrie und der Großgrundbesitzer gegen Demokratie und gesellschaftlichen Fortschritt ist; daß diese Revolution nur deshalb erfolgreich war, weil die Struktur und die

488 Vgl. Peter Intelmann, Chancen und Dilemma des politischen Reformismus, Baden-Baden 1996, S. 30 f. Bei dieser Studie handelt es sich um eine hervorragende, mit wissenschaftlicher Akkuratesse erstellte Werkbiografie Franz Neumanns, die für dieses »Negativporträt« eine zentrale Bedeutung besitzt. Vgl. auch zu Neumann grundsätzlich den informativen, hoffentlich bald auf den neuesten Forschungsstand gebrachten Band: Alfons Söllner, Neumann zur Einführung, Hannover 1982; zudem ders., Franz L. Neumann - Skizzen zu einer intellektuellen und politischen Biographie, in: Franz L. Neumann. Wirtschaft, Staat, Demokratie. Aufsätze 1930-1954, Frankfurt a. M., S. 7-56, sowie ders., Neumann als Archetypus - die Formierung des political scholar im 20. Jahrhundert, in: Matthias Iser/ David Strecker (Hg.), Kritische Theorie der Politik. Franz Neumann-eine Bilanz, Baden-Baden 2002, S. 39-55.

489 Vgl. in diesem Zusammenhang die grundsätzlichen Reflexionen zur Bedeutung der Emigration für Intellektuelle von Franz Neumann in dem Aufsatz Intellektuelle Emigration und Sozialwissenschaft aus dem Jahre 1952, die indes als lebensgeschichtliche Erfahrung von Franz Neumann gelesen werden können, in: Franz L. Neumann. Wirtschaft, Staat, Demokratie. Aufsätze 1930-1954, Frankfurt a. M. 1978, S. 402-423, hier S. 409-415.

490 So Helge Pross in den einleitenden Worten in: Franz Neumann, Demokratischer und autoritärer Staat. Studien zur politischen Theorie, Frankfurt a. M. 1986, S. 9-27, hier S. 11. 
Praxis der Weimarer Verfassung sie begünstigten; daß die Revolution weitgehend durch die Entstehung eines Anti-Staates ermöglicht wurde, den der demokratische Staat duldete, obwohl er geschaffen wurde, um die Demokratie zu zerstören; daß die Sozialdemokratische Partei und die Freien Deutschen Gewerkschaften, die als einzige Kräfte die parlamentarische Demokratie verteidigten, zu schwach waren, um dem $\mathrm{Na-}$ tionalsozialismus zu widerstehen, und daß ihre Schwäche sowohl unvermeidlich wie selbstverschuldet war.

Und an anderer Stelle hieß es wie folgt: »Die deutsche Demokratie hat Selbstmord verübt und ist gleichzeitig ermordet worden. Diese Demokratie ohne Demokraten endete mit der Ernennung Hitlers zum Kanzler am 30. Januar 1933.« ${ }^{491}$

Im Londoner Exil ging es Neumann alsbald darum, die vereinzelten und sozusagen vom Nationalsozialismus politisch geschlagenen Emigranten zusammenzuführen. Die zahlreichen nach England geflohenen Sozialisten und ehemalige Kommunisten - wie zum Beispiel Karl Korsch und Franz Borkenau - gehörten zu einem politisch-theoretischen Diskussionszirkel, der von Neumann ins Leben gerufen wurde. ${ }^{492} \mathrm{Im}$ Wesentlichen widmete er sich allerdings seinem Studium der Politikwissenschaft und Soziologie an der London School of Economics. Durch die Vermittlung von Harold Laski - dem zu diesem Zeitpunkt von der marxistischen Theorie beeinflussten führenden Theoretiker der Labor Party - erhielt er ein Stipendium und weitere Hilfeleistungen jüdischer Organisationen. 1936 schloss er sein Studium mit der Promotionsarbeit The Governance of the Rule of Law. An Investigation into the Relationship between the Political Theories, the Legal System and the Social Background in the Competitive Society bei Laski ab, die unveröffentlicht blieb, aber nichtsdestotrotz für seine Studie Behemoth von fundamentaler Bedeutung sein sollte. ${ }^{493}$ Mittels der Kontakte zu Max Horkheimer

491 Franz L. Neumann, The Decay of German Democracy (dt.: Der Niedergang der deutschen Demokratie), in: Ders., Wirtschaft, Staat, Demokratie. Aufsätze 1930-1954, S. 103-123 (Zitate S. 104 u. 119).

492 Vgl. im Einzelnen Peter Intelmann, Chancen und Dilemma des politischen Reformismus, S. 34 f.

493 Zur Promotionsarbeit schreibt RolfWiggershaus, Die Frankfurter Schule. Geschichte-Theoretische Entwicklung - Politische Bedeutung, München 1991, S. 253 f.: »Das Buch - methodisch vor allem Karl Mannheim, Max Weber und Marx und inhaltlich vor allem Harold Laski verpflichtet - bestand im Wesentlichen aus zwei großen Teilen. Im ersten, ideengeschichtlichen Teil untersuchte Neumann politische Theorien von Thomas von Aquin bis Hegel unter dem Cesichtspunkt, wie sie das Verhältnis zwischen Souveränität des Staates und Freiheit des Individuums sahen. Im zweiten rekonstruierte er am Beispiel Englands und Deutschlands, wie im 19. und 20. Jahrhundert das Verhältnis zwischen Wirtschaftssystem, politischem System und Rechtssystem aussah - geleitet von den Fragen, was sich daraus für die Einschätzung der Rolle des Rechts und für die Chancen einer annähernden Versöhnung von Staatssouveränität und individueller Freiheit lernen ließ.« Und vor dem Hintergrund der neumannschen Ansicht, dass der liberale Rechtsstaat durchaus »progressive Elemente« entwickelt hatte, schrieb Wiggershaus, dass ebendiese »unverzichtbaren Bestandteile eines jeden die Freiheit des Individuums ernst nehmenden Staates bilden mußten: >Generalität des Gesetzes, Unabhängigkeit des Richters, Teilung der Gewalten - dies sind Prinzipien, die die Bedürfnisse des Konkurrenzkapitalismus übersteigen, weil sie die persönliche Freiheit sichern. Sie verhüllen zwar die wirkliche Macht einer bestimmten Gesellschaftsschicht und sie machen die Austauschprozesse berechenbar, aber sie schaffen eben persönliche Freiheit und Sicherheit auch für den Armen. [...] Wir wiederholen noch einmal, daß alle drei Funktionen in der Epoche des Konkurrenzkapitalismus realisiert worden sind. Wichtig ist auch, sie voneinander zu unterscheiden. Hält man sie nämlich nicht auseinander und erblickt in der Gesetzesgeneralität nichts als ein Erfordernis der kapitalistischen 
avancierte Neumann im Laufe des Jahres 1936 zum Mitarbeiter des von Frankfurt nach New York übergesiedelten Instituts für Sozialforschung. Im Rahmen der »Institutsvorlesungen « hielt er im Winter 1936/37 in der Extension Division der Columbia University Vorlesungen über den totalitären Staat, die bei den Studenten großen Anklang fanden. Neben mehreren Rezensionen in der Zeitschrift für Sozialforschung veröffentlichte er in den kommenden Jahren in ebenjener "Institutszeitschrift« ein »Konzentrat« (Rolf Wiggershaus) seiner Dissertation unter dem Titel Der Funktionswandel des Gesetzes im Recht der bürgerlichen Gesellschaft (1937) sowie den Aufsatz Types of Natural Law in den Studies in Philosophy and Social Sciences (1940). Im September 1939 wurde er mit der Entscheidung des Leiters des Institutes für Sozialforschung, ${ }^{494}$ Max Horkheimer, konfrontiert, dass er die Forschungsstätte zum 1. Oktober 1940 verlassen müsse. ${ }^{495}$

$\mathrm{Zu}$ diesem Zeitpunkt arbeitete Neumann fast ausschließlich an der politischen, ökonomischen, sozialen und ideologischen Strukturanlayse des Nationalsozialismus. In seinem zuerst 1942 dann in erweiterter Form 1944 in New York erschienenen und nach einer Gestalt aus der jüdischen Mythologie benannten Buch Behemoth interpretierte er das NS-Regime als einen zur »Herrschaft der Gesetzlosigkeit« mutierenden "Unstaat«, in dem unmittelbar nach Hitlers Machtübernahme Staat und NSDAP um die Durchsetzung ihres Totalitätsanspruchs kämpften. ${ }^{496}$ Neumann zufolge hatte sich

Ökonomie, dann natürlich muß man mit Carl Schmitt folgern, daß alle diese Prinzipien, die Ceneralität des Cesetzes, die richterliche Unabhängigkeit, die Teilung der Cewalten, vernichtet werden müssen, wenn der Kapitalismus zugrunde geht.< [...] Neumanns Buch schien so zu besagen: Hoffnung gab es nur, wenn noch einmal so etwas wie ein liberaler Rechtsstaat zustande kam mit einer herrschenden Schicht, die vor der faschistischen Lösung zurückschreckte. Ähnlich wie sein Lehrer Lasky blieb Neumann, obwohl nun auf der Ebene gesellschaftstheoretischer Analyse marxistisch denkend, politisch ein Reformist, der alles von einer besseren Politik der Organisationen der Arbeiterbewegung unter wiederhergestellten rechtsstaatlichen Bedingungen erhoffte."

$494 \mathrm{Zu}$ den unterschiedlichen Forschungsansätzen und Analysen in Bezug auf den Nationalsozialismus und mithin zur Kontextualisierung von Neumanns Behemoth im »Horkheimer-Institut«vgl. Helmut Dubiel/Alfons Söllner, Die Nationalsozialismusforschung des Instituts für Sozialforschung - ihre wissenschaftliche Stellung und ihre gegenwärtige Bedeutung, in: Dies. (Hg.), Wirtschaft, Recht und Staat im Nationalsozialismus, Frankfurt a. M. 1984, S. 7-31.

495 Vgl. Wiggershaus, Die Frankfurter Schule, S. 257.

496 Vgl. ebd., S. 75 ff. und 90-93 (Zitat S. 16). Ich stütze mich bei der Interpretation der Studie auf Cert Schäfer, Franz Neumanns Behemoth und die heutige Faschismusdiskusion, in: Ebd., S. 663-776, sowie besonders auf Armin Nolzen, Franz Leopold Neumanns Behemoth. Ein vergessener Klassiker der NS-Forschung, in: Zeithistorische Forschungen/Studies in Contemporary History 1 (2004), S. 150153. 
aus diesem Gegensatz von »totalitärem Staat (Leviathan) und »totalitärer Bewegung" (Behemoth) mit Beginn des Zweiten Weltkrieges eine neue Gesellschaftsform herausgebildet, in der die herrschenden Gruppen die Bevölkerung qua Propaganda und Gewalt »direkt kontrollierten - ohne die Vermittlung durch den wenigstens rationalen, bisher als Staat bekannten Zwangsapparat « ${ }^{497}$.

Neumanns Analyse des NS-Systems ging davon aus, dass die einheitliche Staatsgewalt seit 1933 Schritt für Schritt zerfallen sei und stattdessen vier Gruppen unterund miteinander um die Macht im Staate konkurrierten. Als neue, die Bevölkerung unmittelbar beherrschende Machtblöcke hätten sich NSDAP, Ministerialbürokratie, Wehrmacht und Wirtschaft herauskristallisiert. ${ }^{498}$ Neumann zufolge waren diese Herrschaftsträger in sich souverän und der Prozess der politischen Willensbildung im Behemoth basierte auf ungeregelten Absprachen zwischen den einzelnen Akteuren. Alle notwendigen politischen Maßnahmen beruhten auf stets neu auszuhandelnden Kompromissen zwischen den Funktionseliten der NSDAP, der Ministerialbürokratie, der Wehrmacht und der Wirtschaft. Hierbei bildete die NSDAP das dynamischste Element. Sie stellte für Neumann eine riesige Bürokratie dar und besaß die Funktion, den personellen »Erneuerungsprozess der herrschenden Klasse « zu koordinieren. ${ }^{499} \mathrm{Zu}$ diesem Zweck habe sich die NSDAP nach und nach völlig unabhängig von der staatlichen Verwaltung gemacht und verfolgte das Ziel, den öffentlichen Dienst mit zuverlässigen Parteigängern zu durchdringen und gleichzeitig die Beamtenschaft im Sinne der NS-Ideologie zu indoktrinieren. ${ }^{500}$ Für Neumann war die NSDAP 1944 zur bestimmenden politischen Kraft des NS-Staats geworden. ${ }^{501}$

Im Rahmen seiner Theorie des auf vier Säulen basierenden NS-Regimes rückte Neumann indes das Wirtschaftssystem in den Mittelpunkt seiner Betrachtungen, das für ihn weniger ideologisch als von seiner größtmöglichen Effizienz und Produktivität für die Kriegsführung beherrscht wurde. Er beschrieb es als eine Monopol- und Befehlswirtschaft, in der die Selbstverwaltung der Wirtschaft nicht angetastet worden sei. ${ }^{502}$ »Im Gegenteil: Die Ministerialbürokratie habe die ökonomische Selbstverwaltung zusätzlich gestärkt, indem sie die Bildung von Monopolen in der Wirtschaft durch gesetzliche Eingriffe begünstigt habe. $\aleph^{503}$ Der »totalitäre Monopolkapitalismus«, wie Neumann das nationalsozialistische Wirtschaftssystem charakterisierte, war hierbei funktionaler Bestandteil des auf systematische Machterweiterung ausgerichteten Regimes: »Die Triebkraft des ökonomischen Systems « war indes der »aggressive, imperialistische expansionistische Geist des deutschen Großkapitals «. ${ }^{504}$ Das nationalsozialistische Deutschland musste für Neumann »Eroberungszüge durchführen«, damit die vier herrschenden Gruppen »die Ernte einfahren können«. Das war für ihn das »einzige, die herrschende Klasse zusammenhaltende Band «. ${ }^{505}$

497 Vgl. Neumann, Behemoth, S. 505-530 (Zitat S. 543).

498 Vgl. ebd., S. 541-550.

499 Vgl. ebd., S. 107-113, 434-440 und 461 ff. (Zitat S. 461).

500 Vgl. ebd., S. 90-104, 430-434 und 440-444.

501 Vgl. ebd., S. 562-572.

502 Vgl.zum Folgenden ebd., S. 269-422.

503 Nolzen, Franz Leopold Neumanns »Behemoth«, S. 152.

504 Neumann, Behemoth, S. 415.

505 Ebd., S. 459. 
Einerseits erschien also in Neumanns Interpretation das NS-Regime nicht als geschlossener, monolitischer, totalitärer Block, andererseits richtete er den Blick auch auf die »charismatische Herrschaft« (Max Weber) und mithin auf die soziale Beziehung des »Führers« zur deutschen Bevölkerung, wobei er weniger auf die Propaganda und Inszenierung des Charismatikers als auf die Erwartungen und Hoffnungen derjenigen abhob, die an Hitler glaubten. Denn: »Die charismatische Macht des Führers ist kein bloßes Trugbild - niemand kann bezweifeln, daß Millionen an sie glauben. ${ }^{506}$

Die Studie von Neumann stieß insbesondere in den wissenschaftlichen Kreisen der Vereinigten Staaten auf breite Zustimmung ${ }^{507}$ und verschaffte dem Autor »internationales Renommee ${ }^{508}$ Im Zweiten Weltkrieg begann dann für den ausgewiesenen Kenner des NS-Herrschaftssystems und für Deutschlandfragen - unter anderem mit seinen ebenfalls aus dem Institut für Sozialforschung ausgeschiedenen Freunden Otto Kirchheimer, Arcadius Gurland und Herbert Marcuse - eine mehrjährige Zusammenarbeit mit der US-amerikanischen Administration. ${ }^{509}$ Von 1942 bis 1945 arbeitete Neumann - dessen Mutter dem nationalsozialistischen Terror zum Opfer fiel und 1940 im Konzentrationslager Theresienstadt ums Leben $\mathrm{kam}^{510}$ - zuerst im neugeschaffenen Office of Strategic Services (OSS) in Washington und agierte nach der deutschen Kapitulation als Leiter der German Research Section im amerikanischen Außenministerium. ${ }^{511}$ Seine zentrale Aufgabe bestand darin, den amerikanischen Besatzungsapparat auf die zukünftige komplexe Demokratisierungspolitik in Deutschland vorzubereiten. Das primäre Ziel von Neumann, der Ende 1943 die amerikanische Staatsbürgerschaft erhalten hatte, bestand zu diesem Zeitpunkt darin, einen durchschlagenden umfassenden Entnazifizierungsprozess in Gang zu bringen, der auch eine konkrete wirtschaftliche, soziale und politische - man könnte sagen - gesamtgesellschaftliche Strukturveränderung Deutschlands in Angriff nehmen sollte. Eine ganz entscheidende Rolle spielte Neumann sowohl bei den Vorbereitungen als auch bei der Entwicklung des konzeptionellen Gerüsts für die 13 Nürnberger Prozesse als Mitarbeiter des amerikanischen Stabes des Hauptanklägers Robert H. Jackson. ${ }^{512}$

506 Ebd., S. $116 \mathrm{f}$.

507 Zur Rezeption des Behemoth vgl. Intelmann, Chancen und Dilemma des politischen Reformismus, S. 46f. (Anm. 237), sowie Gert Schäfer, Franz Neumanns Behemoth und die heutige Faschismusdiskussion, in: Neumann, Behemoth, S. 663-776, hier S. 716, Anm. 3 mit näheren Ausführungen zu der Rezension von Ernst Fraenkel.

508 So Söllner, Franz L. Neumann, S. 10.

509 Vgl. hierzu bes. Rainer Erd (Hg.), Reform und Resignation. Gespräche über Franz L. Neumann, Frankfurt a. M. 1985, S. 153-171. Vgl. grundsätzlich immer noch für die im Laufe der nationalsozialistischen Gewaltherrschaft aus Deutschland emigrierten politischen Intellektuellen und Wissenschaftler, die danach für den US-amerikanischen Staat arbeiteten: Söllner, Zur Archäologie der Demokratie in Deutschland.

510 Intelmann, Chancen und Dilemma des politischen Reformismus, S. 20.

511 Vgl. hierzu im Einzelnen die Ausführungen in Kap. I.1.5.

512 Vgl. hierzu ausführlich Joachim Perels, Franz L. Neumanns Beitrag zur Konzipierung der Nürnberger Prozesse, in: Iser/Strecker, Kritische Theorie der Politik, S. 83-94, der indes auch feststellt, dass Neumanns Einfluss und der seiner OSS-Mitarbeiter auf die jeweilige Prozessführung nicht allzu groß war, da deren Arbeiten sowie analytische Studien für die an historisch-empirische Einzelbelegen ausgerichtete Prozessvorbereitung weniger geeignet waren (ebd., S. 85 f.). Vgl. auch Taylor, Die Nürnberger Prozesse, S. 69. 
Für die in wissenschaftlicher, genuin politisch-praktischer bzw. politisch-moralischer Absicht geschriebenen Arbeiten von Neumann als Leiter der »Deutschlandabteilung« im State Department direkt nach dem alliierten Sieg über den deutschen »Unstaat«, dessen Zusammenbruch für ihn - vor allem angesichts der für den Verfasser des Behemoth quasi systemimmanenten Massenverbrechen - ausschließlich durch eine militärische Intervention von außen erfolgen konnte, stand folgende Frage im Zentrum: "Wie ist eine demokratische Lebensform in Deutschland wiederherstellbar, und welches sind die Bedingungen ihrer erfolgreichen Etablierung? ${ }^{513}$ Vor dem Hintergrund der historisch-analytischen Erkenntnisse über den Untergang der Weimarer Republik, der Machtetablierung sowie der destruktiven Machtakkumulation des NS-Systems, deren Ursachen für Neumann zuallererst in den ökonomischen, sozialen und staatlichen Strukturbedingungen, aber auch in antidemokratischen Vorstellungen der meisten deutschen Staatsbürger lagen und die natürlich nicht »über Nacht« verschwunden waren, kritisierte er sehr bald die amerikanische Besatzungspolitik in Deutschland. Die zum Teil vehemente Kritik zielte neben dem Protest gegenüber dem Wiederaufleben des »Nationalismus « und »Chauvinismus« in Deutschland in erster Linie auf den mangelnden Willen der US-Militärregierung, die in Gang gebrachte umfassende gesamtgesellschaftliche Entnazifizierung auch zum Abschluss zu bringen. ${ }^{514}$

Gleichwohl beteiligte sich Neumann auch nach seinem offiziellen Ausscheiden aus dem amerikanischen Staatsdienst im Jahre 1947 aktiv und konkret an der »Reeducation«bzw. an der Demokratisierung und »Verwestlichung« Deutschlands. Obwohl ihn seine Lehrtätigkeit als »ordentlicher Professor« an der Columbia Universität in New York, deren Ruf ihn 1950 erreichte, ${ }^{515}$ eminent beanspruchte, ${ }^{516}$ engagierte sich der »dreifache Außenseiter ("als Emigrant, linker Intellektueller und Jude«) ${ }^{517}$ auf seinen zahlreichen Deutschlandbesuchen zum Beispiel angesichts der zugespitzten Situation Berlins in der Hochphase des Kalten Krieges im Juni 1950 für den »Kongreß für kulturelle Freiheit«, um durch seine Anwesenheit sowie Mitarbeit in diesen »heißen Tagen«

513 Söllner, Franz L. Neumann, S. 28.

514 Vgl. hierzu folgende Aufsätze: Die Umerziehung der Deutschen und das Dilemma des Wiederaufbaus (1947), S. 290-308, Militärregierung und Wiederbelebung der Demokratie in Deutschland (1948), S. 309326, und Deutsche Demokratie (1950), S. 327-372, in: Neumann, Wirtschaft, Staat, Demokratie.

515 Helge Pross, Einleitung, in: Neumann, Demokratischer und autoritärer Staat, S. 15.

516 Neumann hatte nicht nur zahlreiche Studenten, sondern zudem einige Schüler, die später sehr bekannte Wissenschaftler wurden. Zu einem seiner sicherlich prominentesten Schüler zählte der prominente Holocaust-Forscher Raul Hilberg, der unter dem Einfluss des Behemoth und der anfänglichen Hilfe seines Doktorvaters 1961 in den Vereinigten Staaten seine bahnbrechende Untersuchung The destruction of the European Jews veröffentlichte. Siehe hierzu die entsprechenden Passagen im Gespräch zwischen Raul Hilberg und Alfons Söllner, Das Schweigen zum Sprechen bringen. Ein Cespräch über Franz Neumann und die Entwicklung der Holocaust-Forschung, in: Dan Diner (Hg.), Zivilisationsbruch. Denken nach Auschwitz, Frankfurt a. M. 1988, S. 175-200. Vgl. auch den Hinweis von Intelmann, Chancen und Dilemma des politischen Reformismus, S. 53 (Anm. 278), der neben Raul Hilberg noch Fritz Stern, Julian Franklin, Kurt L. Shell, Herbert Deane, David Kettler, Peter Gay und Jeane Kirkpatrick als Schüler von Franz Neumann ausweist.

517 Hubertus Buchstein, Politikwissenschaft und Demokratie. Wissenschaftskonzeption und Demokratietheorie sozialdemokratischer Nachkriegspolitologen in Berlin, Baden-Baden 1992, S. 297. 
gegen jedwedes totalitäres Herrschaftsmodell Stellung zu beziehen. ${ }^{518}$ Allerdings hatte Lasky aufgrund des kapitalismuskritischen (Forschungs-)Ansatzes kein Interesse daran, dass Neumann in dem Periodikum veröffentlicht. ${ }^{519}$ So blieb der öffentliche Auftritt von Neumann in Berlin 1950 die einzige Mitarbeit für den »Kongreß für kulturelle Freiheit« bzw. für den Monat.

Allerdings bleibt festzuhalten, dass sich Neumann sehr wohl als politischer Intellektueller und Theoretiker mit den »Ideologien des Kalten Krieges« beschäftigte und hierbei das nicht zuletzt in Deutschland grassierende manichäische Weltbild vom apriorischen Gegensatz zwischen den westlichen und östlichen Systemen, anders gesagt: zwischen freiheitlich-parlamentarischer Demokratie und östlichem Totalitarismus, beleuchtete. Nach Neumanns Dafürhalten gab es den sogenannten Dritten Weg zwischen den beiden "Großblöcken", d.h. zwischen dem kapitalistischen und dem kommunistischen Gesellschaftssystem. Wie seinerzeit Richard Löwenthal ${ }^{520}$ sah auch er die gesellschaftliche Alternative in einem "genuin westeuropäischen Sozialismus", also in einem demokratischen Rechtsstaat, in dem die individuellen Freiheitsrechte gesichert waren, indes in einem sozialistisch organisierten Wirtschaftssystem. ${ }^{521}$

Gleichwohl verstummte der ehemalige Mitarbeiter vom New Yorker Institut für Sozialforschung angesichts der tatsächlichen Verhältnisse in der Sowjetunion unter Stalin nicht. Im Gegensatz beispielsweise zu Horkheimer und Adorno - die weit in die 1930er-Jahre hinein mit der marxistisch-kommunistischen Idee und mit dem Zentrum der antibürgerlichen Revolution in Moskau sympathisierten - stellte der Linksintellektuelle und politische Theoretiker Neumann in den 1950er-Jahren, als in Deutschland die Totalitarismustheorie vor allem durch Arendts Doppelanalyse des Nationalsozialismus und des Sowjetkommunismus Hochkonjunktur hatte, in seinen Aufsätzen Theorie der Diktatur (der Aufsatz bestach auch durch den Versuch, die totalitären Bewegungen mithilfe sozialpsychologischer Kategorien Freuds zu interpretieren) und Ökonomie und Politik im zwanzigsten Jahrhundert auch einen konkreten Vergleich zwischen dem nationalsozialistischen und dem sowjetkommunistischen Herrschaftsregime im Lichte der Genese der modernen westlichen Industriesysteme an. ${ }^{522}$

518 So nahm Neumann, wie gesagt, auf einer öffentlichen Diskussionsveranstaltung zu »politische[n] Themen« teil, die im Rahmen des »Kongresses für kulturelle Freiheit«im Studentenhaus der Technischen Universität stattfand und von der Zeitschrift Der Monat organisiert wurde. Auf der Veranstaltung zum sogenannten Thema »Adolf Hitler und sein Reich« hielt »Professor Franz L. Neumann von der Columbia-Universität« den einleitenden Beitrag, der »in einer historischen Analyse den allgemeinen und den besonderen deutschen Tendenzen zum Totalitarismus, namentlich unter soziologischen Aspekten «nachging, woraufhin ebendiese Veranstaltung »in ihrem Diskussionsteil einen unerwarteten Verlauf«nahm, so die Mitteilung der Zeitschrift. Denn:»Es entspann sich die vielleicht erregteste Auseinandersetzung aller Kongreßveranstaltungen, die jedoch nur der kritischen Beleuchtung des Themas zugute kam.«Der Monat 2 (1950), H. 22/23, S. 474 f.

519 Mitteilung von Lasky in einem Cespräch mit dem Verfasser im Juni 2000 in Berlin.

520 Vgl. hierzu Paul Sering (d. i. Richard Löwenthal), Jenseits des Kapitalismus, Lauf bei Nürnberg 1946 sowie die entsprechenden Ausführungen im »Löwenthal-Porträt« in Kap. II.4.2.

521 So Söllner, Franz L. Neumann, S. 32.

522 Vgl. den indes nicht endgültig fertiggestellten Aufsatz Notizen zur Theorie der Diktatur (1957), in: Franz Neumann, Demokratischer und autoritärer Staat. Beiträge zur Soziologie der Politik, Frankfurt a. M. 1967, S. 147-170, sowie »Ökonomie und Politik im zwanzigsten Jahrhundert« (1955), in: Ebd., S. 171-183. Vgl. auch ders., Ansätze zur Untersuchung politischer Macht, in: Ebd., S. 58-75. Zur Ein- 
$\mathrm{Zu}$ diesem Zeitpunkt arbeitete Neumann angesichts der destruktiven Entwicklung in den europäischen Ländern im 20. Jahrhundert mit ihren totalitären, faschistischen und autoritären Herrschaftsregimen an der Ausformulierung einer zeitgenössischen kritischen Gesellschaftstheorie unter Berücksichtigung der politischen Freiheit und der politischen Theorie, genauer gesagt: unter Einbeziehung der Wissenschaft der Politik in der Demokratie ${ }^{523}$ In der Auseinandersetzung mit den großen Problemen »unserer Zeit« spielten für Neumann die Intellektuellen eine zentrale Rolle, und zwar gerade angesichts des Problems von »Angst und Politik«, d.h. der realen »Möglichkeit der Pöbelherrschaft «. ${ }^{524} \mathrm{Da}$ für ihn nämlich diese Tendenzen den demokratisch verfassten Gesellschaftssystemen inhärent waren, bestand für Neumann die Pflicht, diesen Gefahren entgegenzutreten und sowohl als Intellektueller wie auch als Wissenschaftler die politische Freiheit zu verteidigen, um nicht, wie die »deutschen Intellektuellen und Akademiker« in den 1930er-Jahren, einen neuerlichen »Verrat« (Julien Benda) zu begehen. ${ }^{525}$

Vor dem Hintergrund der Etablierung einer freien Wissenschaft sowie einer nach angelsächsischer Tradition ausgerichteten Politikwissenschaft in Deutschland, zumal als kritische Gesellschaftstheorie neumannscher Provenienz, hatte der Freund von Otto Suhr entscheidenden Anteil an der Gründung der Freien Universität, der Deutschen Hochschule für Politik und nicht zuletzt des Instituts für politische Wissenschaft in der geteilten Stadt Berlin. ${ }^{526}$ Mittels der Bemühungen von Neumann entschloss sich auch sein Freund aus den »frühen Tagen «, Fraenkel, seinen aus existenzieller, d.h. totalitärer Erfahrung entsprungenen Entschluss, eingedenk der nationalsozialistischen Judenvernichtung zu Lebzeiten niemals mehr nach Deutschland zurückzukehren, aufzugeben. Insofern zählte es auch zu den Verdiensten von Neumann, einen der renommiertesten emigrierten Politikwissenschaftler an die neuen Berliner Forschungseinrichtungen zu holen. ${ }^{527}$

Abgesehen davon, dass Lasky aufgrund des kapitalismuskritischen (Forschungs-) Ansatzes von Neumann kein Interesse daran hatte, dass dieser in dem Zeitschriftenorgan veröffentlicht, dürften weitere Gründe existiert haben. Da sich der kapitalismuskritische Forschungsansatz zweifelsohne auch in Neumanns insbesondere historische und sozioökonomische sowie politisch-ideologische Aspekte verbindende Analyse des Untergangs der Weimarer Republik und der Genese der nationalsozialistischen Machteroberung einerseits und des nationalsozialistischen Herrschaftssystems andererseits niederschlug, wird auch deshalb eine Mitarbeit aus der Sicht der Zeit-

ordnung des »Spätwerks« von Neumann im Kontext der klassischen Totalitarismustheorien von Arendt sowie Friedrich und Brzezinski siehe Alfons Söllner, Geschichte und Herrschaft. Studien zur materialistischen Sozialwissenschaft 1929-1942, Frankfurt a. M. 1979, S. 202-205.

523 Vgl. hierzu insbesondere den Aufsatz aus dem Jahre 1950 Franz Neumann, Die Wissenschaft der Politik in der Demokratie, in: Ders., Wirtschaft, Staat, Demokratie, S. 373-392. Vgl. auch unter diesem Aspekt ders., Intellektuelle Emigration und Sozialwissenschaft (1952), in: Ebd., S. 402-423.

524 Ders., Angst und Politik (1954), in: Neumann, Demokratischer und autoritärer Staat, S. 184-214.

525 Ders., Intellektuelle und politische Freiheit (1955), in: Ebd., S. 215-229, hier S. 225.

526 Vgl. Intelmann, Chancen und Dilemma des politischen Reformismus, S. 55.

527 Vgl. hierzu im Einzelnen ebd., S. $57 \mathrm{ff}$. 
schriftenredaktion ausgeschlossen gewesen sein. ${ }^{528}$ Ebenso aufgrund von Neumanns Reflexionen und Einschätzungen zur konkreten amerikanischen Besatzungspolitik in Deutschland, die er, wie gesagt, wegen des mangelnden Willens der US-Militärregierung, die in Gang gebrachte umfassende gesamtgesellschaftliche Entnazifizierung auch zum Abschluss zu bringen, vehement kritisierte. Zudem muss auch berücksichtigt werden, dass die analytische Brillanz von Neumann, die er insbesondere im Behemoth unter Beweis stellte, im Hinblick auf das stalinistische Herrschaftssystem vor 1945 und speziell auf die Politik Stalins nach dem Ende des Zweiten Weltkrieges nicht in Anwendung kam. Anders gesagt: Im Vergleich zu dem auch lebensgeschichtlich erworbenen Analyseniveau der »Sowjetexperten « wie zum Beispiel der Renegaten Borkenau, Koestler und Löwenthal konnte Neumann - obwohl der Stalinismus für ihn kein Anathema war - nicht mithalten. Auch dieser Umstand muss sicherlich berücksichtigt werden bei der Frage, warum Neumann nicht für den Monat geschrieben hat. Ob es hierbei irgendwelche Einmischungen der US-Militärregierung, der Pariser Zentrale des »Kongresses für kulturelle Freiheit« oder der CIA gab, d. h. vor allem, ob Neumann im Monat infolge eines Verbotes nicht veröffentlichen durfte, oder ob sich Lasky aus »freien Stücken« gegen eine Zusammenarbeit mit Neumann aussprach, kann erst nach einer Auswertung der in den Archiven in den Vereinigten Staaten liegenden Unterlagen und Dokumente zur Geschichte der Zeitschrift Der Monat beantwortet werden. ${ }^{529}$

528 Ich werde am Ende der »Analyse des Nationalsozialismus im Monat« (Kap. IV.1) in dem Abschnitt IV.1.11 noch einmal darauf zurückkommen.

529 Ich komme auf diesen Punkt im Schlussteil dieser Arbeit zurück. 